\title{
Laser-Plasma Harmonics with High-Contrast Pulses and Designed Prepulses
}

\author{
R.S. Marjoribanks, ${ }^{1}$ L. Zhao, ${ }^{1}$ F.W. Budnik, ${ }^{1}$ G. Kulcsár, ${ }^{1}$ \\ A. Vitcu, ${ }^{1}$ H. Higaki, ${ }^{1 *}$ R. Wagner, ${ }^{2}$ A. Maksimchuk, ${ }^{2}$ D. Umstadter, ${ }^{2}$ \\ S.P. Le Blanc, ${ }^{3}$ and M.C. Downer ${ }^{3}$
}

(1) Department of Physics, University of Toronto

60 St. George St.; Toronto, Ontario; Canada MSS IA7

(2) Center for Ultrafast Optical Science, University of Michigan 2200 Bonisteel Blvd.; Ann Arbor, MI 48109

(3) Department of Physics, University of Texas at Austin, Austin, Texas 78712

\begin{abstract}
One aspect of the complexity of mid- and high-harmonic generation in highintensity laser-plasma interactions is that nonlinear hydrodynamics is virtually always folded together with the nonlinear optical conversion process. We have partly dissected this issue in picosecond and subpicosecond interactions with preformed plasma gradients, imaging and spectrally resolving low- and mid-order harmonics. We describe spatial breakup of the picosecond beam in preformed plasmas, concomitant broadening and breakup of the harmonic spectrum, presumably through self-phase modulation, together with data on the sensitivity of harmonics production efficiency to the gradient or extent of preformed plasma. Lastly, we show preliminary data of regular Stokes-like and anti-Stokes-like satellites to the harmonics, accompanied by modification of the forward-scattered beam.
\end{abstract}

\section{INTRODUCTION}

With the development of ultra-intense, high-contrast, sub-picosecond lasers, midand high-harmonic generation from thin, near-solid-density plasmas has become an productive topic in recent years, complementary to nanosecond laser-plasma harmonics research dating back nearly twenty years $(1,2,3,4)$. Several experimental studies have been reported recently $(5,6,7)$, different models have been developed $(8,9,10,11)$, and new mechanisms suggested in this regime, largely based on particle-in-cell simulations.

One of the important issues in mid- and high-harmonic generation from solid target is the laser pulse contrast, because of its effect on the electron density profile. In earlier $\mathrm{CO}_{2}$ experiments, using nanosecond pulses, the ponderomotive force of the laser pulse was seen to steepen the plasma density profile significantly (12). But in the picosecond laser/solid target interactions, the ponderomotive steepening is less effective because of the short interaction time. Therefore the plasma preformed by the pedestal or prepulse

* current address: Graduate School of Human and Environmental Studies, Kyoto University, Sakyoku, Kyoto, 606-01 Japan. 
becomes very important. Although this preplasma issue in harmonic generation has been mentioned before, no systematic experiment on this effect has been reported.

It would be interesting and useful to characterize the optical participation of electrons without at the same time grossly modifying the ion density gradients during irradiation, principally by ponderomotive steepening. To this end, we have conducted a series of experiments in which a plasma gradient is prepared by a separate laser prepulse, and the effect of gradient on the harmonic production from an intense main pulse is examined.

\section{EXPERIMENTS}

\section{Laser Systems Used}

Two laser systems were used in this work, whose main parameters are summarized in Table 1. The FCM-CPA laser at the University of Toronto is a Nd:glass-based chirped-pulse amplification laser system, based on gratings-only expansion and compression of high-contrast 1 -ps seed pulses which have energy greater than $1 \mu \mathrm{J}$ per pulse; these pulses are directly produced in a feedback-controlled Nd:glass oscillator (13). In the final output of the system, the intrinsic pulse-to-pedestal contrast is better than $5 \times 10^{7}$. Under some conditions, a Pockels-cell-leakage prepulse is observed in the output, at $10^{-4}$ of peak and $1.5 \mathrm{~ns}$ ahead of the main pulse. To eliminate these prepulses, we placed a saturable-absorber dye-cell in the final output line, producing a final 'clean' pulse contrast greater than $10^{10}$. In experiments, this system delivered 1 ps pulses with energies to $200 \mathrm{~mJ}$, focused at the target to intensities up to $10^{17} \mathrm{~W} \mathrm{~cm}^{-2}$.

TABLE 1: Summary of laser parameters.

\begin{tabular}{|c|c|c|c|c|c|}
\hline Laser system & $\lambda(\mu \mathrm{m})$ & $\mathrm{I}_{\max }\left(\mathrm{W} \mathrm{cm} \mathrm{cm}^{-2}\right)$ & $\tau(\mathrm{ps})$ & Contrast & Method \\
\hline \hline FCM-CPA & 1.053 & $1 \times 10^{17}$ & 1.1 & $>10^{10}$ & dye-cell \\
\hline $\mathrm{T}^{3}$ & 0.527 & $1 \times 10^{18}$ & 0.4 & $>10^{10}$ & crystal \\
\hline
\end{tabular}

The $\mathrm{T}^{3}$ laser at CUOS, University of Michigan, is a hybrid Ti:sapphire and Nd:glass CPA laser system operating at a wavelength of $1.053 \mu \mathrm{m}$, capable of producing 400 -fs pulses with energies up to $3 \mathrm{~J}$ (14). Intrinsic pulse contrast is $\sim 10^{5}$; in order to increase it, the infrared pulse was converted to its second harmonic $(\lambda=527 \mathrm{~nm})$ using a 4-mm thick type-I KD ${ }^{*} \mathrm{P}$ crystal (15). Four harmonic beam-splitters and a BG39 glass filter were used after the crystal to filter out the infrared component in the laser beam. The contrast for the green pulse was estimated to be better than $10^{10}$.

\section{Picosecond, $\lambda=1 \mu \mathrm{m}$ Experiments}

First, a series of experiments on the second harmonic generation were made with the FCM-CPA laser system, concentrating on the effect of preplasma produced by small prepulses. Polished flat silicon targets were used. With the ppolarized laser incident at $35^{\circ}$ from the target normal, the $2 \omega$ signal was collected in the specular direction. We compared the

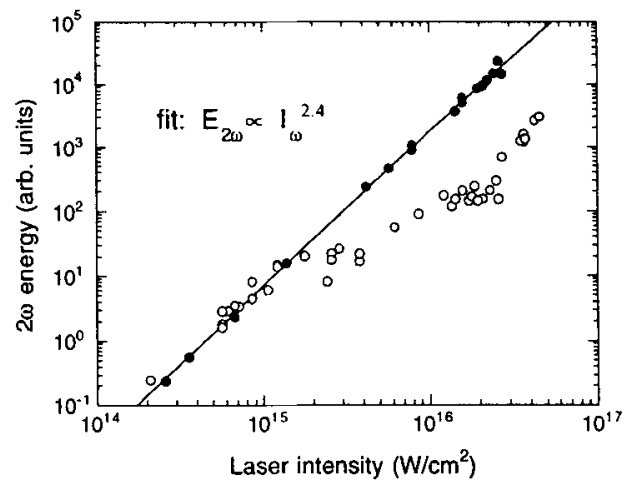

Fig. 1: $2 \omega$ yield from clean pulses (solid) and with fixed-fraction prepulse (open circles). 
$2 \omega$ yield under two experimental conditions: 'clean pulse' (with dye-cell in) and 'intrinsic pulse' (with dye-cell out). Figure 1 shows the laser intensity scaling of the $2 \omega$ energies measured with the intrinsic pulse and with the dye-cell-cleaned pulses. No difference was observed while the laser intensity was below $2 \times 10^{15}$ $\mathrm{W} \mathrm{cm}-2$. As the laser intensity passed $2 \times 10^{15} \mathrm{~W} \mathrm{~cm}^{-2}$, the collected $2 \omega$ energy from the intrinsic-contrast pulses began to saturate, presumably as preplasma was formed.

To examine this yield loss, the angular distribution of the second harmonic was measured at a laser irradiance of $2 \times 10^{16} \mathrm{~W} \mathrm{~cm}^{-2}$ under these two pulsecontrast conditions. The $2 \omega$ angular distribution spread out and its intensity dropped with prepulse present (Fig. 2). After correcting for the increased solid angle, the total yields are almost the same with and without preformed plasma.
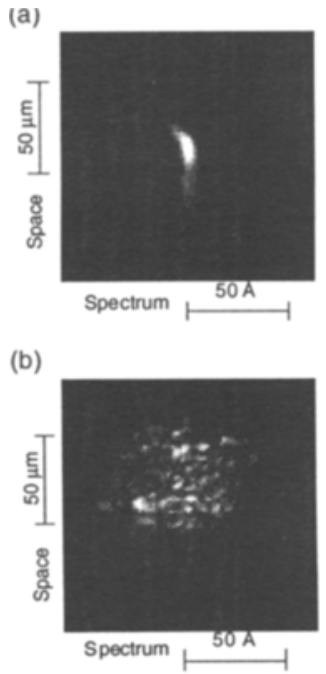
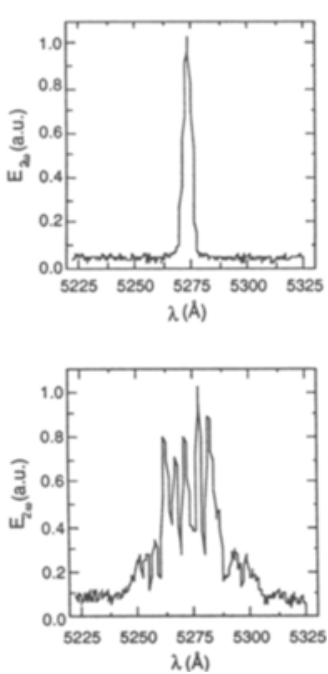

Fig. 3: Spatially resolved $2 \omega$ spectrum: (a) with clean pulse; (b) with $10^{-4}$ prepulse. At left is a spectrum (resolved horizontally) for a slit-image of the plasma (vertically), from center of the laser focus; at right is a lineout of the spectrum through centre. using a partially amplified beam. Harmonic spectra were recorded using a 1-m Seya-Namioka type VUV spectrometer (McPherson) equipped with a 1200 lines/mm grating. The laser focal spot on target was positioned on the Rowland circle and served as would an entrance slit for the spectrometer. At the output, a microchannel plate intensifier (Galileo) was used as the detector, lens-coupled to a 16-bit CCD camera (Photometrics) located outside the vacuum chamber.
Secondly, the details of the spatial and spectral structure of the harmonic source were examined, using a $30 x$ image of the harmonic source relayed from the focal spot to an imaging spectrograph to give simultaneous spatial and spectral resolution. The results shown in Figure 3 illustrate the complex spatial and spectral changes which accompany beam breakup and self-phase modulation in the preformed plasma of Figs. 1 and 2.

\section{$350 \mathrm{fs}, \lambda=0.53 \mu \mathrm{m}$ Experiments}

For higher-harmonic experiments, the CUOS $\mathrm{T}^{3}$ laser system was used. An $\mathrm{f} / 3.023-\mathrm{cm}$ focal length, off-axis $\left(15^{\circ}\right)$ parabolic mirror was used to focus the 43-mm diameter laser beam onto the target at an incidence angle of $60^{\circ}$ from normal. The focal spot was measured Fig. 2: $2 \omega$ yield: clean pulses (solid dots); fixed-
fraction prepulse (circles). Deviation seen in Fig. 1 follows this spread to larger solid-angle, inferred from modest changes in the angle of incidence. 


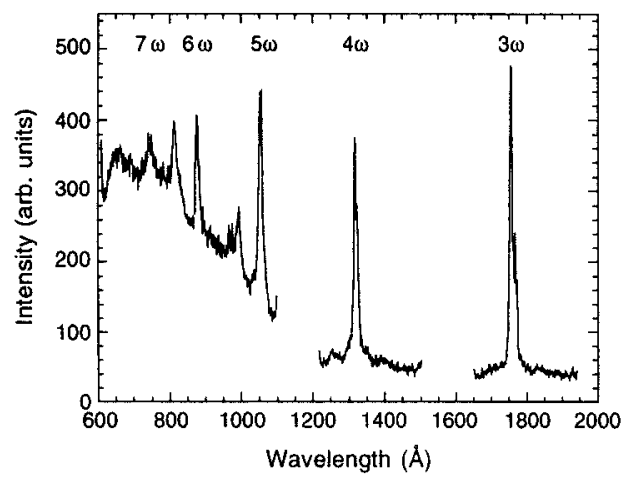

Fig. 4: Typical time-integrated harmonic spectrum from Si target, $\mathrm{I} \sim 3.2 \times 10^{17} \mathrm{~W} \mathrm{~cm}^{-2}$.
Several flat targets of various atomicnumber materials (beryllium, $\mathrm{CH}$, silicon, aluminum, nickel, gold) were used in this experiment. Harmonic spectra from all these targets exhibited similar features. Figure 4 shows a typical time-integrated harmonic composite spectrum recorded from a silicon target irradiated in $p$ polarization at a laser intensity of $3.2 \times$ $10^{17} \mathrm{~W} \mathrm{~cm}^{-2}$. Harmonics from 3 rd to 7 th, of both odd and even orders, can be easily identified, sitting on top of a broad Z-dependent plasma recombination background (Fig. 4).

By rotating a half-wave plate located after the doubling crystal, the $3 \omega$ yield from a beryllium target was measured both with p- and s-polarized incident laser pulses. With irradiances up to $1 \times 10^{18} \mathrm{~W} \mathrm{~cm} \mathrm{~cm}^{-2}$ on target, we observed clear $3 \omega$ signal $(\mathrm{S} / \mathrm{N} \sim 30$ ) for p-polarized irradiation but no $3 \omega$ signal for s-polarized irradiation (Fig. 5). Since there is no analyzing polarizer for the harmonic output in the current arrangement, we cannot draw clear conclusions about the polarization of the harmonics generated. It is likely that there is some polarizationselection inherent in diffraction from the grating of the spectrograph. If we assume that the p-polarized incident irradiation produced p-polarized harmonics (11) and that the grating efficiency of the spectrometer is 5 times greater for ppolarization than for s-polarization, we can conclude from our results that with spolarized irradiation any $3 \omega$ yield is: a) if p-polarized, no more than 1/30th of that from the p-polarized laser pulse, and b) if s-polarized, 1/6th $(5 / 30)$ of that from the ppolarized laser pulse.

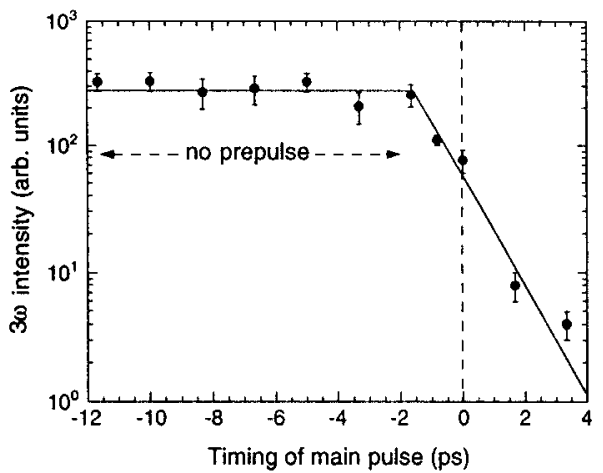

Fig. 6: Relative efficiency of $3 \omega$ from flat $\mathrm{Si}$ vs. prepulse/main pulse delay. Prepulse is $5 \%$ of the main pulse, which is $5 \times 10^{17} \mathrm{~W} \mathrm{~cm}^{-2}$.

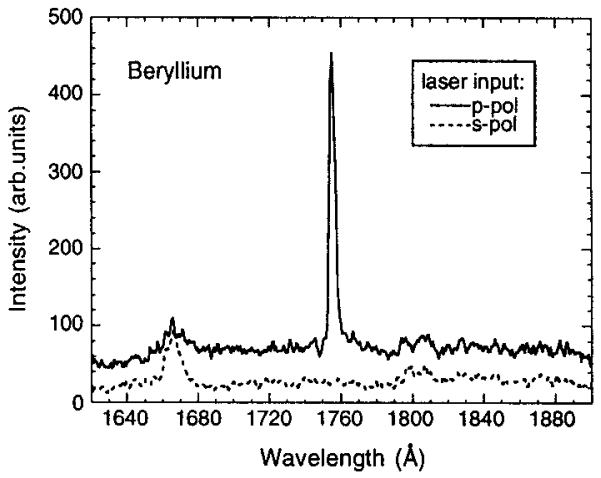

Although a satisfactory model of the relation between harmonic generation and plasma-scalelength is still lacking for these laser-plasmas, it is generally believed that the efficiency of harmonic generation will vary with the gradient of the density profile (16). Experimentally, the gradient of the plasma density profile can be controlled by adding a deliberate prepulse at a controllable time before the main pulse arrives. In this way, the effect of density gradient on harmonic generation can be studied quantitatively.

After the compressor, we split out a small part of the infrared beam, frequencydoubled it in a crystal similar to that used in
Fig 5: Spectra recorded using p-polarized laser pulses (solid), and s-polarized pulses (dashed). 
the main beam, and then co-propagated it with the infrared beam. The focussed intensity and spot-size of the prepulse were controlled by a an adjustable diaphragm. In this experiment, the main pulse intensity was $5 \times 10^{17} \mathrm{~W} \mathrm{~cm}-2$, and the prepulse intensity was set to be $2.5 \times 10^{16} \mathrm{~W} \mathrm{~cm}^{-2}-$ about $5 \%$ of the main pulse. The timing between peaks of these two orthogonally polarized green pulses was determined with a resolution of \pm 70 fs by frequency-domain interferometry. Figure 6 shows $3 \omega$ yield from separate shots onto a flat Si target, collected in the specular direction, as the delay between the main pulse and the small prepulse was increased. It can be seen that the harmonic efficiency starts to decrease once the prepulse moves ahead of the main pulse, and drops quickly by two orders of magnitude for $5 \%$ prepulses arriving 3 ps ahead of the main pulse.

Previously, it has been established that such intensities do produce motion of the critical density surface, near the peak of the pulse (17) and presumably some steepening occurs over $200 \mathrm{fs}$ or so. A sense of the dependence of third-harmonic efficiency on the scalelength initially seen by a generating pulse can nonetheless be inferred from hydrodynamic modelling of the plasma expansion, following the prepulse. For each delay in Figure 6, the scalelength of the evolving plasma was calculated using the $1-$ D hydrocode MEDUSA (18), and the new correspondence plotted in Figure 7: as the scalength changes from about $0.1 \lambda$ to about

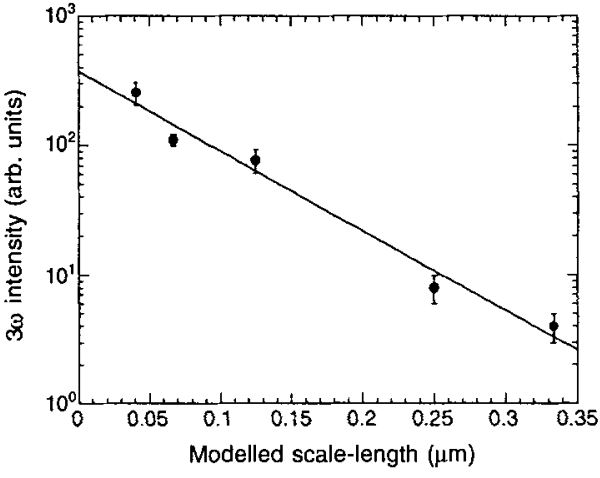

Fig. 7: Efficiency of $3 \omega$ vs. MEDUSA-modelled scalelength, from prepulse delays in Fig. 6. $0.66 \lambda$, the third-harmonic efficiency drops by two orders of magnitude.

In resolving the structure of harmonic lines at higher irradiances, we have observed the development of novel satellite lines, both red- and blue-shifted, which appear to have a regular Stokes- and anti-Stokes-like structure. These lines appeared around each of the 3rd-6th harmonics, apparently simultaneously but appreciably after the appearance of the harmonics themselves. The appearance intensity of these satellites, in our geometry, was mid-10 $17 \mathrm{~W} \mathrm{~cm}^{-2}$. As the irradiance was increased to $1 \times 10^{18} \mathrm{~W} \mathrm{~cm}^{-2}$, we observed the sequential appearance of three such peaks: first a red-shifted peak, then on blue-shifted peak, then an additional red-shifted peak, each stepped in frequency by the same increment. The satellites were repeatable and spectrally narrow; in a few cases,

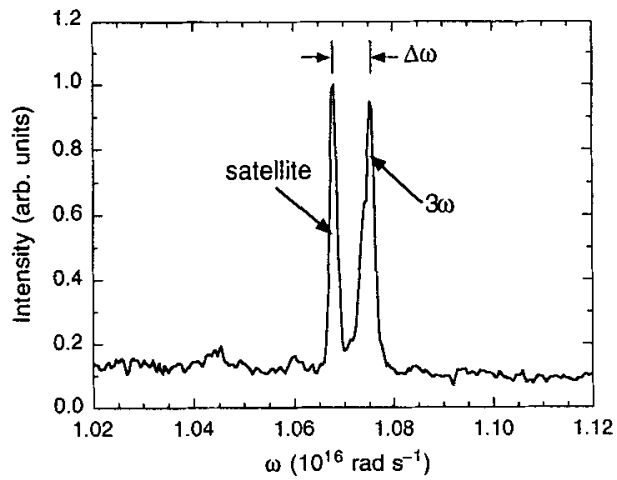

Fig. 8: The appearance of the first red-shifted satellite to $3 \omega$ from a parylene-N target $(\mathrm{CH}) ; \mathrm{I} \sim$ $4 \times 10^{17} \mathrm{~W} \mathrm{~cm}^{-2}, \Delta \omega=7.4 \times 10^{13} \mathrm{rad} \mathrm{s}^{-1}$. the line on the longer-wavelength side was as intense as the harmonic line itself (Fig. 8).

These lines appeared for different atomic-number materials: $\mathrm{Be}, \mathrm{CH}, \mathrm{Al}$, and $\mathrm{Si}$, but were not seen under any of our conditions for the higher- $\mathrm{Z}$ elements, $\mathrm{Ni}$ and $\mathrm{Au}$. For $\mathrm{CH}$ targets, the frequency step between satellites was $\sim 7.4 \times 10^{13}$ rad $\mathrm{s}^{-1}$; initial analysis suggests that this shift may be weakly Z-dependent, with a possible $10 \%$ difference between $\mathrm{Be}$ and $\mathrm{Si}$.

At a sensitivity of $10^{-4}$ of incident intensity, we detected no backscattered light. However, the spectrum of forward- 
scattered laser light showed sudden line-centre depletion and broadening exactly upon the appearance of the satellite features.

We do not at present have a satisfactory explanation for these features, but we note that the frequency step between satellites corresponds approximately to the resonant electron plasma wave frequency scaled by the square root of the ratio of electron and ion masses and presumed charge-to-mass ratio, $c f$., an associated ion plasma wave.

\section{CONCLUSIONS}

In using very high contrast pulses to which we have added back deliberate, controlled prepulses, we have partly separated the contributions of nonlinear hydrodynamics and nonlinear optics in the generation low- and mid-order laser-plasma harmonics. We have shown how previously noted spectral breakup of harmonic spectra follows from preformed plasmas caused by small prepulses, characterized the effect of prepulse in spreading harmonic emission over large solid angles, and quantified the sensitivity of harmonic generation efficiency to the gradient of preformed plasma. Finally, we have reported new observations of regular Stokes-like and anti-Stokes-like satellite features accompanying the harmonics, possibly resulting from the participation of ions near critical.

\section{ACKNOWLEDGMENTS}

The authors gratefully acknowledge the support of several agencies: RSM: the Natural Sciences and Engineering Research Council of Canada, Photonics Research Ontario, and the Fellows Program, Center for Ultrafast Optical Science, University of Michigan; DPU: grants NSF PHY 972661 and NSF STC PHY 8920108; SPL, MCD: partial support of DOE grant DE-FG03-97ER54439, Robert Welch Foundation grant F1038, and a Faculty Research Assignment from the University of Texas.

\section{REFERENCES}

1. Burnett, N.H., Baldis, H.A., Richardson, M.C., Enright, G.D., Appl. Phys. Lett. 31, 172 (1977).

2. Carman, R.L, Forslund, D.W., and Kindel, J.M., Phys. Rev. Lett. 46, 29 (1981).

3. Carman, R.L., Rhodes, C.K., and Benjamin, R.F., Phys. Rev. A 24, 2649 (1981).

4. Bezzerides, B., Jones, R.D., and Forslund, D.W., Phys. Rev. Lett. 49, 202 (1982).

5. von der Linde, D., et al., Phys. Rev. A 52, R25 (1995).

6. Kohlweyer, S., et al., Opt. Commun. 117, 431 (1995).

7. Norreys, P.A., et al., Phys. Rev Lett. 76, 1832 (1996).

8. Esarey, E., et al., IEEE Trans. Plasma Sci. 21, 95 (1993).

9. Gibbon, P., Phy. Rev. Lett. 76, 50 (1996).

10. Wilks, S.C., Kruer, W.L., and Mori, W.B., IEEE Trans. Plasma Sci. 21, 120 (1993).

11. Lichters, R., Meyer-ter-Vehn, J., and Pukhov, A., Phys. Plasmas 3, 3425 (1996).

12. Fedosejevs, R, et al., Phys. Rev. Lett. 43, 1664, (1979).

13. Marjoribanks, R.S., et al., Opt. Lett. 18, 361 (1993).

14. Mourou, G., and Umstadter, D., Phys. Fluids B 4, 2315 (1992).

15. Chien, C.Y., et al., Opt. Lett. 20, 353 (1995).

16. Delettrez, J., Bonnaud, G., LLE Review 58, 76 (1994).

17. Liu, X., and Umstadter, D., Phys. Rev. Lett. 69, 1935 (1992).

18. Christiansen, J.P., Ashby, D.E.F.T., and Roberts, K., Comput. Phys. Commun. 7, 271 (1974); Djaoui, A., and Rose, S.J., J. Phys. B 25, 2745 (1992). 